\section{Efeitos da lasalocida sobre a produção de vacas leiteiras em sistema semi-intensivo}

\author{
Lasalocid effects on dairy cows production of in a semi-intensive \\ management system.
}

Correspondence 10:

Carlos de Souza Lucci Departamento de Nutrição e Producào Animal

Faculdade de Medicina

Veterinária e Zootecnia da USP

Caixa Postal 23

Av. Duque de Caxias Norte. 225 13630-970 - Pirassununga - SP. Brasil

e-mail: cslucci@usp.br

1. Pesquisador - Instituto de Zootecnica

2. Médico Veterinário

3. Departamento de Nulriçào e

Producâo Animal

Faculdade de Medicina

Veterinaria e Zootecnia da USP

- SP

\title{
RESUMO
}

Foram estudados os efeitos da lasalocida sobre a produção de leite, teor e percentagem de gordura no leite, variaçōes de peso e condiçōes corporais de 18 vacas leiteras do ecótipo Mantiqueira, com média de 75 dias pós-parto ao inicio do experimento, alimentadas com $1,0 \mathrm{~kg}$ de mistura de concenirados para cada $2,5 \mathrm{~kg}$ de leite produzidos acima dos primeiros 4,0 kg, capim elefante (Pennisetum purpureum, var. Napier) picado, fornecido no cocho, e pastagem de Bracharia brizantha. Utilizou-se um delineamento em blocos casualizados e os tratamentos consistiram na administração diária de 0,200 e $300 \mathrm{mg}$ de lasalocida/ animal. O experimento teve duração de 17 semanas, sendo as quatro primeiras destinadas ao periodo de adaptação. Independente da dose empregada a lasalocida nāo alterou significativamente a produção de leite e a produção de leite corrigida a $4 \%$ de gordura, porém o grupo tratado com $200 \mathrm{mg}$ apresentou maior porcentagem de gordura no leite $(P<0,05)$. Os grupos tratados com 200 e $300 \mathrm{mg}$ apresentaram ganhos de peso, porém não estatisticamente menores em relação aos animais controle, quando os dados foram analisados como percentagem de peso vivo. Não foi observada alteração na condição corporal dos animais.

UNITERMOS: Ionóforos; Lasalocida; Produção de leite; Ruminantes

\section{INTRODUÇÃO}

A lasalocida é um ionóforo poliéter carboxilico, producido pelo Sireprombers lasaliensis (Bergen; Bates;. 1984).

Ao ser comparada com a monesina, apresentada vantagens de maior palatabilidade e menor toxide\% além de pequena ou nenhuma queda na ingestão de alimentos em dictas eom alta energia, conferindo maiores ganhos de peso nessas dictas (Spears ${ }^{15}$. 1990). ()s ionoforos possuem uma série de efeitos sobre a fermentaçào ruminal (Bergen: Bates ${ }^{3}$. 1984), dentre os quais podem ser citados o aumento da produçào de ácido propiônico em detrimento do acético e/ou butírico, diminuição na produção de metano, no consumo de alimentos e na taxa de passagem ruminal. alteraçiòo da digestibilidade do alimento, diminuição da degradação protécica da produçào de ácido láctico e da incidência de cimpanismo (Schelling ${ }^{1+}, 1984$ ). Na formaçào dos acidos acético L butírico são produridos gases ricos em encrgia, como o metano, eliminado pela crutaçiòo. Por outro lado, o acido propiônico constitui-se na forma mais eficiente de aproveitar energia dos alimentos. Outro efeito da lasolocida é o de restringir as bactérias gram positivas. nào attuando nals gram negativas, que formall acido propionico. Desta forma, os ionoforos possuem a capacidade de aunentar a eliciênciado metabolismo energético e protéco no rímen e no animal, além de diminuir a incidencia de distúrtios digestivos (Bergen; Bates3. 1984).
Os resultados decorrentes da utilizaçào dé ionóforosos prin-cipalmente a monensina. no desempenho de bovinos de corte, foram intensivamente estudados na última decada. (ioodrich a al. ${ }^{\mathrm{x}}$ (1984). em revisão do assunto, observaram aumento de $1.6 \%$ no ganho de peso e diminuiçio de $6,4 \%$ no consumo de alimentos. o que resultou em uma melhoral de $7.5^{\circ}$ \% na conversio alimentar. Entretanto. as informaçōes relativas aos efeitos dessass substancias sobre a produção de leite sáo bastante restritas e nada conclusivas. dado o pequeno número de experimentos è al imensa gama de variáveis que interagem sobre os resultados.

O objetivo deste trabalho loi o de avaliar os efejtos da lasalocida sobre o desempenho produtivo de vacals leiteiras.

\section{MATERIAL E MÉTODOS}

Este experimento foi desenvolvido na Estação Experimental de Zootecnia de Pindamonhangaba, Iocalizada no Vale do Paraiba. São Paulo, com altitudes varriando entre 530 a 550 metros acima do nivel do mar: o clima da regiáo é do tipo ( wa, segunda a classificaçào Köppen. co solo é classificado como Latossolo Vermelho Amarelo (Oto-lase Terraço, segundo o ('NI:PA.

Deroito vacans leiteiras do ecolipo Mantiqueira, com duas ou mais lactaçóes e com periodo médio de 75 dias pós-parto ao inicio do experimento. foram separadas em três blocos formados de 
acordo com a produçäo de leite e distribuidas aleatoriamente aos tratamentos compostos pela administração de $0.200 \mathrm{mg}$ e $300 \mathrm{mg}$ de lasalocida por animal e por diat. () delincamento estatistico utilizado loi o de blocos inteiramente casualizados (comes? $1985)$.

() animais foram submetidos a um periodo de adaptaçio de 4 semanas e a um experimental de 13. realizados durante os meses de janciro a abril de 1995. Durante o periodo de adaptação, os animais que perlenciam aos grupos aos quais seria fornecida a lasalocida receberam $200 \mathrm{mg}$ do produto animalidia. As vacas permaneceram em regime semi-intensivo, vindo para o estábulo somente no momento das ordenhas e para receberem alimentaçõo no cocho, permanecendo o tempo restante em pastagem de Brachiaria brizantha. Os animais cram ordenhados as 6:00 a as It:(0) horas e recebiam alimentaçào no cocho em duas partes: uma imediatamente apos a ordenha. no periodo da manhà. ea outra antes da ordenha da tarcke

Por ocasiào das ordenhas cra ministrada, por animal e por dia. mistura concentrada, na base de $1.0 \mathrm{~kg}$ para canda $2.5 \mathrm{~kg}$ de leite produrido acima dos 4.0 primeiros quilogramas; $10.0 \mathrm{~kg}$ de capim

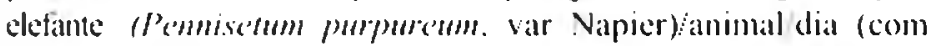
base na matéria original). 60 g de mistura mincralianimal dia eo produto teste $(0,200)$ e $300 \mathrm{mg}$ de lasalocidatanimal dia). A quantidade de concentrado oferecida loi corregida mensalmente atratrés do fator de persistencia na queda da produção de leite. utilizando-se. para tal, o menor fator medio. dentro de um tratamento. para a correção dos demais

A mistura concentrada consistiu de $50.0 \%$ de grãos de milho moidos. $30.0 \%$ de farelo de soja. $15.0 \%$ de farelo de trigo. $3.0 \%$ de calcário calcílico, $1.0 \%$ de fosfato bicáleico, $0,5 \%$ de sal comum e $0.5 \%$ de premix mineral. A concentraçăo por quilograma de premix era (68.93 g de fösforo, $173.60 \mathrm{~g}$ de cálcio. $150.77 \mathrm{~g} \mathrm{de} \mathrm{sódio.}$ $3.95 \mathrm{~g}$ de cuxolice, $4.19 \mathrm{~g}$ de zinco, $2.10 \mathrm{~g}$ de ferro, $839 .(0) \mathrm{mg}$ de cobre, $25,31 \mathrm{mg}$ de selenio, $50.63 \mathrm{mg}$ de iodo, $16,78 \mathrm{mg}$ de cobalts c (0.69 me de fǘr (miximo). A mistura mineral ministrada a pale. na base de 60 ganimal dia. continha $57.14 \%$ de losfato bicácico, $28.57^{\circ}$ o de sal comum a $14.29^{\circ}$ o do premix descrito anteriormente As quantidades ingeridas de calciofóstoro foram do aproximmadimente 30 g ( a 2 : $20 \mathrm{~g}$ P. sem contar a contribuiçáo dos pastos. loi realizada uma amostragem da mistura concentrada, na saricla fo misturador. durante a preparaçào es sta composiça nutricional apresentou valor de $87.3 \%$ de materia seca. $23.0 \%$ de proteina bruta. $2.7 \%$ de extrato eléreo. $7.7 \%$ de fibra bruta e $6.1 \%$ de materia mineral. na base de materia seca isenta de umidade. As análises bromatologicats foram eletuadas eonforme as normas $\triangle O A C^{-1}(1985)$. A raçào foi balanceada considerando-se os dados do.$N R(" 11(1989)$ para proteina. energia, cálcio e fösoro.

Produçós de leite foram medidas semanal mente cas análises dos teores de gordura, a cada duas semanas. empregando-se o teste de (ierber. () anmain foram pesados e alvaliades quanto a condiçao corporal quin/enalmente. sempre no mesmo horário, ats 8:(0) horas da manha. A condiçào corporal foi classificada cm umal cscala de 1 a 5 . sendo a nota 1 usada para animal muito magro e 5. muito gordo.

()s dados foram submetidos à análise de variância pelo procedimento (iI M do Statistical Analysis System (SAS ${ }^{12}$. 1985) Quando o efeito de tratamento foi significativo (leste F). als médias foram comparadas pelo Teste de Tukey (P)<0.05).

\section{RESULTADOS E DISCUSSÃO}

()s efeitos da lasalocida sobre a produçâo de lecite e gordura encontram-sc na Tab. 1

Tabela 1

Efeitos da lasalocida sobre as produções de leite $(\mathrm{PL})$, produções de leite corrigidas $(P L C)$, produçōes de gordura (PG) e porcentagens de gordura (\%G). Pindamonhangaba - SP, Jan. a Abr., 1995.

\begin{tabular}{lcccc}
\hline \multicolumn{5}{c}{ Niveis de lasalocida' } \\
\hline & Controle & $\mathbf{2 0 0} \mathbf{~ m g}$ & $\mathbf{3 0 0} \mathbf{~ m g}$ & C.V.(\%) \\
\hline PL (kg) & $1.146,49$ & $1.162,04$ & $1.133,98$ & 12,50 \\
PLC (kg) & 985,36 & $1.033,57$ & 976,21 & 13,14 \\
$\% G$ & $3,03^{\mathrm{b}}$ & $3,27^{\mathrm{a}}$ & $3,07^{\mathrm{ab}}$ & 5,91 \\
PG ((kg) & 35,00 & 37,94 & 34,70 & 14,16 \\
\hline
\end{tabular}

'Médias dentro da mesma linha com letras sobrescritas diferentes diferem estatisticamente pelo Teste de Tukey $(P<0,05)$.

$\Lambda$ administraçào de 200 ou $300 \mathrm{mg}$ de lassalocida/animal dia na dieta nào alterou significativamente a produçào de lecite corrigida a $4^{\prime \prime}$ de gordura ou a produçióo de gordura durante todo o periodo experimental. Intretanto, observou-se que os animais submetidos ao trattamento com $200 \mathrm{mg}$ de lasalocida demonstraram atumento na porcentigem de gordura no leite $(\mathrm{P}<0,05)$. comparativamente anos animais controle, apesar do grupo submetido a maiores concentraçós do produto $(300 \mathrm{mg}$ animal dia) não $1 \mathrm{crem}$ apresentado os mesmos efeitos. Este aumento. da ordem de $7,9 \%$ na porcentagem de gordura do grupo que recebeu $200 \mathrm{mg}$ de lasal ocida dia em relaçaio ao controle, foi responsável pelo acréscimo (nào significallivo) de $8.4 \%$ e $4,9 \%$ na produção total de gordura c na produção total de leite corrigido, respectivamente.

Fin contraste, Johnson e' al ${ }^{4}$ ( 1988$)$ observaram diminuição de $20 \% .13 \% .27 \%$ a $9 \%$ na produção de leite corrigida a $4 \%$ de gordura. porcentagem de gordura no leite. produçio de gordura e de proteina no leite, respectivamente. em um experimento com duraça de 12 semanas, porém com niveis de lasalocida bastante superiores (aproximadamente $550 \mathrm{mg}$ animal dia). Im um experimento com duração de 21 dias. Dye "l al." (1988) nào observaram alteraçào na produção de leitc. mas relataram diminuiçào linear da porcentagem de gordura láctea com o aumento dos niveis de lasalocida na dieta. Ao realizarem experimentos tambem de curta duraçio (ate 30 dias) com a monensina, Brown; Hoguct (1985) e Sauter of al..$^{13}$ (1989) registraram diminuição de 5 a $15 \%$ e de 10 a $13 \%$ da porcentagem de gordura. respectivamente. Brown: Hoguet (1985) observaram ainda aumento de $10 \%$ porcentagem de proteina láctea em animais submetidos a restição alimentar. Ao utilizarem o mesmo produto em vacas leiteiras, submetidas exclusivamente a regime de pastejo durante It semanas, I. ynch ef al. "1" $(1990)$ ) observaram aumentos significativos de 7 e 8\% na produçäo de leite e proteina do leile. respectivamente, mas nào verificaram alteraçices dos teores de gordura.

Por outro lado. os resultados deste experimento sio mais compativeis com os obtidos por Beede at al? (1986). Weiss; Amiet ${ }^{17}(1990)$ e ('hristensen a al ${ }^{5}(1994)$ ). (que não observaram alteraçòes significativas na produçào ou composição do leite ao administrar lasalocida na dicta de vacas leiteiras durante $f$. 1 te 10 
semanas, respectivamente. Entretanto. Weiss: Amiet ${ }^{17}(1990)$ e Christensen ef al. ${ }^{5}(1994)$ registraram melhoras de $10 \%$ " a a $16 \%$ e de 9\% a 12". nas eficiencias energetica e de produção do keite, explicando o fato pela diminuição da ingestão de matiria seca observada em ambos os experimentes. apesar dos dados observados por Weiss: Amicl ${ }^{17}$ (1990) terem sido significativos somente durante as duas primeiras semanas do experimento. No nosso estudo nào foi possível medir o consumo. já que os animais permaneceram em sistema semi-intensivo.

tixceto para o experimento de Lynch e' al. ${ }^{11}$ (1990), todas as referênciass citadas apresentaram proporção de concentrados na dieta de 35 a 50 " ".", cujos valores são semellantes aos estimados para o presente caso.

Os efeitos da lasalocida sobre os ganhos de peso e condiçòcs fisicas dos animais encontram-se na Tab. 2.

\section{Tabela 2}

Efeitos da lasalocida sobre os ganhos de peso em kg/animal (GP), ganhos de peso como porcentagens dos pesos por dia (GPD), condiçōes corporais médias (CCM) e variações das condições corporais (VCC). Pindamonhangaba - SP, Jan. a Abr., 1995.

\begin{tabular}{lcccc}
\hline & \multicolumn{4}{c}{ Niveis de lasalocida } \\
\hline & Controle & 200 $\mathbf{~ m g}$ & $\mathbf{3 0 0} \mathbf{~ m g}$ & C.V. (\%) \\
\hline GP $(\mathrm{kg})$ & 32,67 & 19,00 & 20,50 & 55,36 \\
GPD $(\%$ PV/dia) & 0,08 & 0,04 & 0,04 & 59,56 \\
CCM1 & 3,25 & 3,47 & 3,23 & 10,63 \\
VCC & $+0,42$ & $+0,25$ & $+0,17$ & 110,83 \\
\hline
\end{tabular}

${ }^{1}$ Classificação: 1 - muito magra, 5 - muito gorda

()s animais tratados com 200 ou $300 \mathrm{mg}$ de lasalocida dia demonstraram ganhos de peso e variação na condição corporal bastante inferiores quando comparados ao grupo controle lintretanto, essa diferença somente foi detectada a um nivel de significancia de $13 \%$, pelo Teste de Tukey, quando o ganho foi expresso como porcentagem de peso vivo por dia, provavelmente devido a grande variabilidade da amosiragem. que apresentou coelicientes de variaçào de $55.59 \%$ e $110 \%$. para o ganho de peso. ganho de peso como porcentagem do peso vivo por dia c variação nal condiçào corporal. respectivamente.

Esses dados foram adicionalmente comparados através do uso de contrastes ortogonais, grupo controle versus demais (contraste 1) e grupo tratado com $200 \mathrm{mg}$ versus $300 \mathrm{mg}$ (contraste 2 ). Atrav és do contraste 1 foi possivel observar que os animais tralados com 200 ou $300 \mathrm{mg}$ de lasalocidadia demonstraram tendencia em apresentar menores ganhos de peso, no decorrer de todo o experimento, em relação ans animais controle (P-0,06) e essa diferença foi estallisticamente significativa quando o ganho de pesor foi expresso como porcentagem de peso vivo e por dia (P-0.05).

Neste contexto, nossos dados parecem estar discordantes com os obtidos por Dye et al. (1988), Johnson "l al." (1988). Weiss; Amiet ${ }^{17}(1990)$ e Christensen ef al (1994), ao utilizarem a lasalocida, e Brown; I Iogue (1985), Satuer e' al. ${ }^{12}(1989)$ e Lynch et al." (1990), com a monensina, já que nenhum desses trabalhos demonstrou alteraçòes sobre o ganho de peso com a utilização de ionóforos na dicta de vacas leiteiras em lactação. Da mesma forma que são discordes aos obtidos com vacias de corte, lambém em laclaçio (Sprotl e' al. "', 1988).

\section{CONCLUSÕES}

Nas condiçōes do presente estudo, as seguintes conclusòes podem ser extraidas:

1) A lasalocida não influenciou a produção de leite. nem a de leite corrigido para 4\% de gordura. em vacas com produçào alo redor de $15 \mathrm{~kg}$ de leite por dia.

2) A lasalocida, ao nível de 200 mg por animal e por dia, aumentou os leores de gordura do leite em relação ao tralamento controle.

3) A lasalocida diminuiu os ganhos de peso em vacas em lactaçào. quando os mesmos foram expressos em porcentagens dos pesos vivos.

\section{Agradecimentos}

Os autores agradecem ao D). Sérgio (arlo Franco Morgulis da I:mpresa Minerthal Produtos Agropecuários L.cdal. e ao Dr. Antonio Rubens ( hagas L ima da Empresa Produtos Roche ()uimicos - Farmacêuticos S.A. pelo apoio téenico é financeiro, que propiciaram a execução deste traballo.

\section{SUMMARY}

The effects of lasalocid over milk, milk fat content, live weight and body condition, were analysed on 18 "Mantiqueira" cows, 75 days after parturition in the begining of the trial. Cows were fed $1 \mathrm{~kg}$ concentrate mixture for each $2,5 \mathrm{~kg}$ of milk produced over the first $4.0 \mathrm{~kg}$ of milk, chopped elephant grass (Pennisetum purpureum, Napier var), and Brachiaria brizantha pasture. A randomized block design was used with 0,200 or $300 \mathrm{mg}$ of lasalocid/animal/day as treatments. The experimental period lasted 17 weeks, and first four weeks were used as an adaptation period to the ionophore. Lasalocid had no effects on milk or fat corrected milk production, but the $200 \mathrm{mg}$ lasalocid group presented higher milk fat percentages $(P<0.05)$. The 200 and $300 \mathrm{mg}$ lasalocid treatments groups showed lower (but not statistically) live weight gain related to control, when the data were analysed as percentage of live weight. There was no variation on cows body condition. 


\section{REFERÊNCIAS BIBLIOGRÁFICAS}

1- AOAC - ASSO IAIION OH OHIC IAL ANALYTICAL CHIMISTS, Official methods of analysis. 1.1.ed. Washington, A.O.A.C. I985. 114Ip.

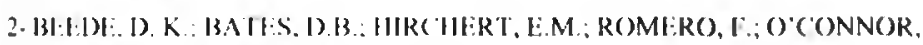

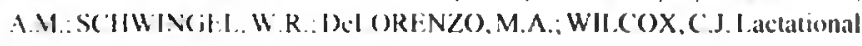
pertirmance of mudactation Holstcin cows fed lasialocid. Journal of A nimal Science. 2:6.3, p.417, 1986. Supplement 1.

3- BI:R(il:V. W. (i.: BATI:S D ) B. Ionophores: their effect on production efficiency and mode of acuon. Juurnal of Animal Science. v.58, n.6. p. 1465 . I984.

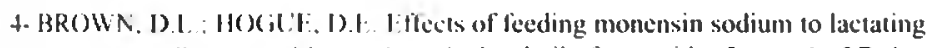
goats: milk tomposition and ruminal volatilc fatty acids. Journal of Dairy

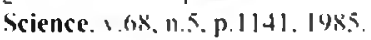

5-(IIRISIINSI:N, I). ., WIIEOMIIIR, R.I), SHENTON,H.T.; BOWMAN, B.R.;

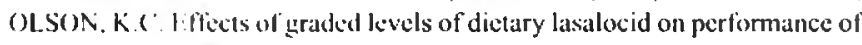
I lolstcin cous durng warly lactaltion. Journal of Animal Science. v. 72, . . 121. 194) S. Supplaturent 2.

6. DVT. B.I... A.MOS II.I... FRO ISC IIIEL, M.A. Influcence of lasalocid on rumen motabolucs. milh production. Imolh composition and digestibility in lac-tating cous Vutrition Reports International. v.38, n.1, p. 101, 1988.

7. GoMI:S. I'P. ( urso de estatistica experimental. I I.ed. Piracicaba. Nobel I:S.11.0. 148.5. 3K+ p.

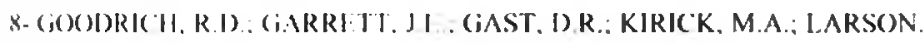
1).A.: MIISKI:, J.C Influence of monensin on the performance of cattle. Journal of Animal Science. 1.5x, n.6. p. 1484.1984.

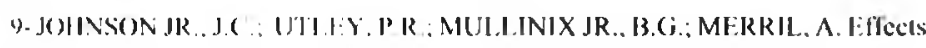
of adding fitt and lasalocid to dicts of dairy cows. Journal of Dairy Science, 1.71. 11.8, p.2151.198x.
10- LYNCH, G.A.; HUNT, M.K:; McCUTCH:ON, S.N. A note on the effect of monensin sodium administered by intranuminal controlled-relcase devices on productivity of dairy cows at pasture Aninal Production. v.51, 11.2. p.418-21, 1990 .

II- NRC - Vutricnt Requirements of I)airy ('attle. National academy of science. Washington. National Academy P'ress, 1989.

12- SAS INSTITUTE: SAS user's guide: statistics. 5.ed. Cary. 1985.

13- SAUER, F.I).: KRAMI:R, J.K.(j.: ( $A$ NTWI:LL, W.J. Anticetogenic effect of monensin in the carly lactancy. Journal of Uairy Science, v.72. n.2. p.436. 1989.

14- SCIILLLINC;, G.T., Monensin mode of action in the rumen. Journal of Animal Science, v.58, n.6, p.1518. 1984.

15- SPLARS. J.W. Modificadores de fermentação ruminal, In: SIMPÓSIO 1)() (OI.FGIO BRASILFIRO DL: NUTRIC ÄO ANIMAI.. 3. Campinas, São Paulo. Anais. 1990. p. 165.

16- SPROTT, L.R.; GOI:IIRING, T.B.: IBI:VE.RI.Y, J R.: CORAII, L.R. IIffects of ionophores on cow herd production: a review. Journal of Animal Science. v.66, n.6, p.1340, 1988 .

17. WFISS, W. P.; AMIET, 13, A. HAltect ollasialocid on performance of latetating dairy cows. Journal of Dairy Science, $1,73, n, 1, \mathrm{p} .153,1990$.

Recebido para publicação: $17 / 07 / 96$ Aprovado para publicação: 08/08/97 